Eur. J. Clin. Chem. Clin. Biochem.

Vol. 30,1992 , pp. $271-274$

(C) 1992 Walter de Gruyter \& Co.

Berlin · New York

\title{
Age-Related Changes in Cerebrospinal Fluid $\gamma$-Aminobutyric Acid Concentration
}

By Haruhiko Takayama ${ }^{1}$, Norio Ogawa ${ }^{2}$, Mitsutoshi Yamamoto ${ }^{3}$, Masato Asanuma $^{2}$, Hiroshi Hirata ${ }^{1,2}$ and Zensuke Ota ${ }^{1}$

1 Third Department of Internal Medicine

${ }^{2}$ Department of Neuroscience, Okayama University Medical School, Okayama, Japan

${ }^{3}$ Department of Neurology, Kagawa Prefectual Central Hospital, Takamatsu, Japan

(Received October 30, 1991/February 24, 1992)

Summary: The purpose of this study was to clarify the effect of aging on brain $\gamma$-aminobutyric acid metabolism. We measured the cerebrospinal fluid $\gamma$-aminobutyric acid concentration in subjects of various ages, including healthy volunteers and patients without neurological or psychiatric disease. The cerebrospinal fluid $\gamma$ aminobutyric acid concentration was determined by radiolabelled receptor assay using $\left[{ }^{3} \mathrm{H}\right] \gamma$-aminobutyric acid. Cerebrospinal fluid $\gamma$-aminobutyric acid was significantly higher in the control group (20s and $30 \mathrm{~s})$ than in the groups of subjects in their $50 \mathrm{~s}, 60 \mathrm{~s}, 70 \mathrm{~s}$ and $80 \mathrm{~s}$. There was a significant negative correlation between cerebrospinal $\gamma$-aminobutyric acid concentration and age $(p<0.01)$. These data suggest that dysfunction of brain $\gamma$-aminobutyric acid metabolism increases with age, and that the various symptoms caused by abnormal $\gamma$-aminobutyric acid metabolism in the brain are therefore more likely to appear in elderly people.

\section{Introduction}

$\gamma$-Aminobutyric acid is thought to be a major inhibitory neurotransmitter. Biochemical studies have revealed that the content of $\gamma$-aminobutyric acid is much lower than normal in the striatum and substantia nigra of autopsied brains from patients with Huntington's disease (1), and the association between neuropsychiatric diseases and the brain $\gamma$-aminobutyric acid system has been studied $(2,3)$. Radiolabelled receptor assays can be used to observe changes in neurotransmission. Recently, $\gamma$-aminobutyric acid was reliably measured in cerebrospinal fluid by a $\gamma$-aminobutyric acid radiolabelled receptor assay, and it was shown that this value can be used as an index of the function of the brain $\gamma$-aminobutyric acid system. We used the $\gamma$-aminobutyric acid radiolabelled receptor assay and found abnormally low concentrations of cerebrospinal fluid $\gamma$-aminobutyric acid in patients with neuropsychiatric diseases, including presenile dementia, Parkinson's disease, Huntington's disease, olivopontocerebellar atrophy and late cortical cerebellar atrophy
$(4,5)$. Other studies have also shown low cerebrospinal fluid $\gamma$-aminobutyric acid concentrations in Huntington's disease (6) and Alzheimer's disease (7). Cerebrospinal fluid is believed to be formed in the choroid plexus and is in contact with both the brain and the spinal cord. Total cerebrospinal fluid $\gamma$-aminobutyric acid concentrations are related primarily to brain $\gamma$ aminobutyric acid concentrations and are minimally affected by changes in the peripheral $\gamma$-aminobutyric acid concentration (8). Changes in cerebrospinal fluid $\gamma$-aminobutyric acid concentrations may therefore reflect changes in brain $\gamma$-aminobutyric acid metabolism.

As the proportion of aged people in the Japanese population increases, their medical problems are receiving more attention. Among these are neurological disorders, including choreatic movement and dementia. In the present study, we measured cerebrospinal fluid $\gamma$-aminobutyric acid concentrations, using a radiolabelled receptor assay, to determine whether $\gamma$ aminobutyric acid metabolism changes during normal aging. 


\section{Subjects and Methods}

Subjects

The subjects consisted of 55 healthy volunteers and patients without neurological or psychiatric disease $(30$ men and 25 women) who were not receiving drugs that act on the central nervous system. Their ages ranged from 21 to 82 years (mean $\pm \mathrm{SD}, 48.4 \pm 18.9$ ). The age distribution is shown in table 1 . Those in their 20 s and 30 s were used as controls, and were compared with the other subjects.

\section{Procedures}

All subjects were maintained on absolute bed rest, and oral intake was forbidden for the 15 hours before the cerebrospinal fluid sample was taken. Some of the patients were not taking medication. In the others, drug therapy was stopped for at least 14 days before the sample was taken. Lumbar puncture was performed at $9 \mathrm{am}$, in the standard fashion with the patients in the lateral decubitus position. Ten millilitres of cerebrospinal fluid were withdrawn and the final $5 \mathrm{ml}$ was immediately frozen by immersion in a mixture of acetone and solid carbon dioxide, and kept at $-70^{\circ} \mathrm{C}$ until assayed.

\section{Receptor preparation}

For the receptor preparation, crude synaptic membrane was prepared from whole rat brains by a modification of the method of De Robertis et al. (9). Briefly, Sprague-Dawley rats (body weight $200-250 \mathrm{~g}$ ) were decapitated and the whole brains were immediately removed. The tissue was homogenized in 10 volumes of an ice-cold $0.32 \mathrm{~mol} / \mathrm{l}$ sucrose solution in a Brinkman Polytron PT-10 homogenizer for $20 \mathrm{~s}$. The homogenate was centrifuged at $900 \mathrm{~g}$ for $10 \mathrm{~min}$, and the supernatant was further centrifuged at $11500 \mathrm{~g}$ for $20 \mathrm{~min}$. The pellet $\left(\mathrm{P}_{2}\right.$ fraction) was resuspended in 10 volumes of Tris- $\mathrm{HCl}$ buffer $(50 \mathrm{mmol} / \mathrm{l}, \mathrm{pH}$ 7.6) and kept at $-70^{\circ} \mathrm{C}$ until assayed. Before analysis, the receptor preparation was thawed and homogenized with a glass homogenizer in 100 volumes of Tris- $\mathrm{HCl}$ buffer, then centrifuged at $50000 \mathrm{~g}$ for $20 \mathrm{~min}$. The supernatant was discarded and the pellet was resuspended in 100 volumes of Tris-HCl buffer containing $0.5 \mathrm{ml} / 1$ Triton $\mathrm{X}-100$. The suspension was shaken in a water bath at $37^{\circ} \mathrm{C}$ for $30 \mathrm{~min}$, then centrifuged twice at $50000 \mathrm{~g}$ for $20 \mathrm{~min}$. The pellet was resuspended in 20 volumes of Tris- $\mathrm{HCl}$ buffer. This gave a tissue protein concentration of about $0.6 \mathrm{~g} / \mathrm{l}$.

Determination of $\gamma$-aminobutyric acid in cerebrospinal fluid

The cerebrospinal fluid $\gamma$-aminobutyric acid concentration was measured by the radiolabelled receptor assay method of Enna \& Snyder (10) as modified by Kuroda et al. (4), with crude synaptic membranes prepared from the rat brain. In brief, 0.5 $\mathrm{ml}$ of the receptor preparation was placed in a glass tube containing $0.2 \mathrm{ml}$ of various concentrations $(100 \mathrm{pmol} / 1-10$ $\mu \mathrm{mol} / \mathrm{l}$, final concentration) of $\gamma$-aminobutyric acid (for standard curve) or cerebrospinal fluid, $0.2 \mathrm{ml}$ of Tris- $\mathrm{HCl}$ buffer and $0.1 \mathrm{ml}$ of $\left[{ }^{3} \mathrm{H}\right] \gamma$-aminobutyric acid (final concentration 6.4 nmol/l, specific activity $2109 \mathrm{TBq} / \mathrm{mol}(57 \mathrm{Ci} / \mathrm{mmol})$, Amersham). The samples were incubated in ice for $30 \mathrm{~min}$ and the reaction was stopped by filtration through glass fibre filters (Whatman GF/C) under reduced pressure. Finally, each filter was washed twice with $5 \mathrm{ml}$ of cold Tris- $\mathrm{HCl}$ buffer, then placed in a scintillation vial with $10 \mathrm{ml}$ of scintillation fluor. The radioactivity of the filter was counted with an automatic beta counter. Specific binding was obtained by subtracting from the total bound radioactivity the amount not displaced by $1 \mathrm{mmol} / 1$ $\gamma$-aminobutyric acid (blank). All samples were analysed in triplicate. Cerebrospinal fluid $\gamma$-aminobutyric acid concentrations were calculated with the aid of the standard $\gamma$-aminobutyric acid radiolabelled receptor assay curve.
Post hoc Dunnett's multiple comparison test (11) was performed following one-way analysis of variance to compare the cerebrospinal fluid $\gamma$-aminobutyric acid concentrations in controls with those in the other age groups.

All data are expressed as means \pm SEMs.

\section{Results}

Table 1 shows the cerebrospinal fluid $\gamma$-aminobutyric acid concentrations in each age group. The concentrations were significantly lower in subjects who were at least 50 years old than in those who were less than 40 years old.

Tab. 1. Cerebrospinal fluid $\gamma$-aminobutyric acid concentrations in various age groups

\begin{tabular}{lrl}
\hline Age (a) & N & $\begin{array}{l}\text { Cerebrospinal fluid } \gamma \text {-aminobutyric } \\
\text { acid (nmol/l) }\end{array}$ \\
\hline $21-39$ & 20 & $127.5 \pm 9.2$ \\
(control) & & \\
$40-49$ & 9 & $99.9 \pm 15.9$ \\
$50-59$ & 9 & $92.4 \pm 5.5^{*}$ \\
$60-69$ & 7 & $74.1 \pm 9.2^{* *}$ \\
$70-79$ & 7 & $66.0 \pm 8.4^{* *}$ \\
$80-82$ & 3 & $39.1 \pm 7.2^{* *}$ \\
\hline
\end{tabular}

The data are shown as means \pm SEMs.

* $\mathrm{p}<0.01,{ }^{* *} \mathrm{p}<0.001$ compared with the control group $(21-39$ a) by post hoc Dunnett's multiple comparison test following one-way analysis of variance.

Figure 1 shows that there was a significant negative correlation between cerebrospinal fluid $\gamma$-aminobutyric acid concentration and age $(\mathrm{p}<0.01)$. This was true for the group as a whole, and also when the data from the men and the women were analysed separately $(p<0.01)$. The age-related decrease was slightly greater, and the correlation was slightly stronger in women.

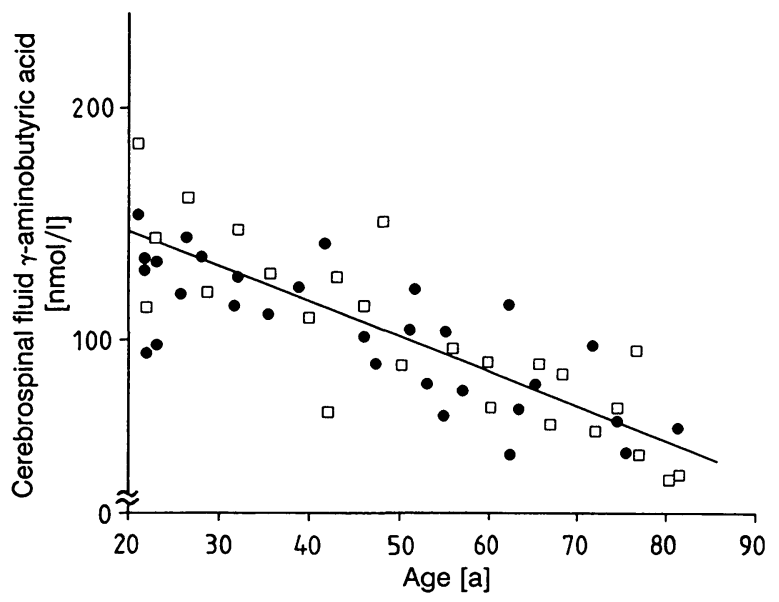

Fig. 1. Relationship between cerebrospinal fluid $\gamma$-aminobutyric acid concentration and age.

Total $(\mathrm{N}=55): \mathrm{y}=-1.41 \mathrm{x}+145$,

- males $(\mathrm{N}=30): \mathrm{y}=-1.19 \mathrm{x}+133$,

$\square$ females $(\mathrm{N}=25)$ : $\mathrm{y}=-1.74 \mathrm{x}+176$.

Linear regression analysis showed a significant negative correlation $(r=-0.81)(p<0.01)$. Significant negative correlations $(p<0.01)$ were also found in men only $(r=-0.79)$ and in women only $(r=-0.88)$. 


\section{Discussion}

$\gamma$-Aminobutyric acid was first detected in neural tissue in $1950(12,13)$. In 1954, Hayashi (14) reported inhibition of seizures after $\gamma$-aminobutyric acid was injected into the cerebral cortex of mammals. This suggested that $\gamma$-aminobutyric acid is an inhibitory neurotransmitter, and subsequent studies have confirmed this. Radiolabelled receptor assays (15) can be used to observe changes in neuro-transmission, and this type of assay has also contributed to studies of $\gamma$-aminobutyric acid receptors. Kuroda (16) has already reported that the dilution curve of cerebrospinal fluid is parallel to the standard $\gamma$-aminobutyric acid radiolabelled receptor assay curve, and that the crossreactivity of glycine, $L$-glutamic acid, and taurine is less than $0.02 \%$. The cerebrospinal fluid $\gamma$-aminobutyric acid concentration could be measured accurately down to $5 \mathrm{nmol} / \mathrm{l}$ by the radiolabelled receptor assay. The existence of rostrocaudal $\gamma$-aminobutyric acid concentration gradients (16) suggests that the cerebrospinal fluid $\gamma$-aminobutyric acid may reflect brain $\gamma$-aminobutyric acid system activity. Although the $\gamma$ aminobutyric acid concentration is about $50 \%$ lower in the cerebrospinal fluid than in plasma, $\gamma$-acetylenic $\gamma$-aminobutyric acid, which is known to increase brain $\gamma$-aminobutyric acid, increased both plasma and cerebrospinal $\gamma$-aminobutyric acid in a dose dependent manner (17). These data indicate that the cerebrospinal fluid $\gamma$-aminobutyric acid originates from the brain and is minimally affected by changes in the peripheral $\gamma$-aminobutyric acid concentration.

We previously reported the cerebrospinal fluid $\gamma$-aminobutyric acid concentrations in patients with various neurological and psychiatric diseases, as measured by radiolabelled receptor assay $(4,5)$. In this study, to evaluate changes in brain $\gamma$-aminobutyric acid metabolism with age, the cerebrospinal fluid $\gamma$-aminobutyric acid concentrations were compared among subjects of various ages, consisting of both normal volunteers and patients without neurological or psychiatric disease. The cerebrospinal fluid $\gamma$-aminobutyric acid concentrations were significantly lower in the subjects who were at least 50 years old than in those who were less than 40 years old (tab. 1). In addition, the cerebrospinal fluid $\gamma$-aminobutyric acid concentration was negatively correlated with age, in both men and

\section{References}

1. Perry, T. L., Hansen, S. \& Kloster, M. (1973) Huntington's chorea: Deficiency of $\gamma$-aminobutyric acid in brain. N. Engl. J. Med. 288, 337-342.

2. Perry, T. L. \& Hansen, S. (1973) Sustained drug-induced elevation of brain GABA in the rat. J. Neurochem. 21, $1167-1175$. women (fig. 1). In particular, the cerebrospinal fluid $\gamma$-aminobutyric acid concentrations of subjects in their 70 s and 80 s were about $50 \%$ and $30 \%$, respectively, of the concentration in the control group.

Hare et al. (18) reported an age-related significant decrease in cerebrospinal fluid $\gamma$-aminobutyric acid concentration among women, but only a slight decrease among men. In this study, computed tomography scans showed relatively slight atrophic changes in the brain (data not shown). Therefore, the decrease in cerebrospinal fluid $\gamma$-aminobutyric acid concentration was probably associated with abnormal $\gamma$-aminobutyric acid metabolism, rather than with a decrease in $\gamma$-aminobutyric acid content due to agerelated atrophy of the cerebral cortex and cerebellum, which contain large amounts of $\gamma$-aminobutyric acid. McGeer (19) reported an age-related decrease in the activity of glutamic acid decarboxylase, which is a $\gamma$ aminobutyric acid synthetase. This might decrease the brain $\gamma$-aminobutyric acid concentration and thus decrease the cerebrospinal fluid $\gamma$-aminobutyric acid concentration. Involuntary movements are frequently seen in the elderly, and memory is gradually impaired. A decrease in the cerebrospinal fluid $\gamma$-aminobutyric acid concentration has been reported in Alzheimer's disease, which is characterized by dementia, in Huntington's disease, which is characterized by involuntary movements, and in Parkinson's disease $(6-8$, 20). In particular, diseases characterized by involuntary movements have been said to be closely associated with $\gamma$-aminobutyric acid metabolism $(6,21)$.

Although the brain $\gamma$-aminobutyric acid system is not the only system involved in the aging process, the present study showed that the cerebrospinal fluid $\gamma$ aminobutyric acid concentration markedly decreased with age. This suggests that brain $\gamma$-aminobutyric acid metabolism is closely associated with various symptoms in the aged, and that the measurement of cerebrospinal fluid $\gamma$-aminobutyric acid can be used to evaluate this association.

\section{Acknowledgement}

This work was supported in part by grants from the Ministry of Education, Science and Culture, and the Ministry of Health and Welfare of the Japanese Government.
3. Perry, T. L., Wright, J. M., Hansen, S. \& MacLeod, P. M: (1979) Isoniazid therapy of Huntington disease. Neurology $29,370-375$.

4. Kuroda, H., Ogawa, N., Yamawaki, Y., Nukina, I., Ofuji, T., Yamamoto, M. \& Otsuki, S. (1982) Cerebrospinal fluid GABA levels in various neurological and psychiatric diseases. J. Neurol. Neurosurg. Psychiat. 45, 257-260. 
5. Ogawa, N., Kuroda, H., Ota, Z., Yamamoto, M. \& Otsuki, S. (1982) Cerebrospinal fluid $\gamma$-aminobutyric acid variations in cerebellar ataxia. Lancet $I I, 215$.

6. Glaeser, B. S., Vogel, W. H., Oleweiler, D. B. \& Hare, T. A. (1975) GABA levels in cerebrospinal fluid of patients with Huntington's chorea: A preliminary report. Biochem. Med. 12, 380-385.

7. Manyam, N. V. B., Katz, L., Hare, T. A., Gerber, J. C. \& Grossman, M. H. (1980) Levels of $\gamma$-aminobutyric acid in cerebrospinal fluid in various neurologic disorders. Arch. Neurol. 37, 352-355.

8. Böhlen, P., Huot, S. \& Palfreyman, M. G. (1979) The relationship between GABA concentrations in brain and cerebrospinal fluid. Brain Res. 167, 297-305.

9. De Robertis, E., Pellegrino de Iraldi, A., Rodriguez de Lores Arnaiz, G. \& Salganicoff, L. (1962) Cholinergic and non-cholinergic nerve endings in rat brain - I. Isolation and subcellular distribution of acetylcholine and acetylcholinesterase. J. Neurochem. 9, 23-35.

10. Enna, S. J. \& Snyder, S. H. (1975) Properties of $\gamma$-aminobutyric acid (GABA) receptor binding in rat brain synaptic membrane fractions. Brain Res. 100, 81-97.

11. Dunnett, C. W. (1964) New tables for multiple comparisons with a control. Biometrics 20, 482-491.

12. Awapara, J., Landua, A. J., Fuerst, R. \& Seale, B. (1950) Free $\gamma$-aminobutyric acid in brain. J. Biol. Chem. 187, 35-39.

13. Udenfriend, S. (1950) Identification of $\gamma$-aminobutyric acid in brain by the isotope derivative method. J. Biol. Chem. $187,65-69$.
14. Hayashi, T. (1954) Effects of sodium glutamate on the nervous system. Keio J. Med. 3, 183-192.

15. Pert, C. B. \& Snyder, S. H. (1973) Opiate receptor: Demonstration in nervous tissue. Science $179,1011-1014$.

16. Kuroda, H. (1983) Gamma-aminobutyric acid (GABA) in cerebrospinal fluid. Acta Med. Okayama 37, 167-177.

17. Löscher, W. (1979) GABA in plasma and cerebrospinal fluid of different species. Effects of $\gamma$-acetylenic GABA, $\gamma$ vinyl GABA and sodium valproate. J. Neurochem. 32, 1587-1591.

18. Hare, T. A., Wood, J. H., Manyam, B. V., Gerner, R. H., Ballenger, J. C. \& Post, R. M. (1982) Central nervous system $\gamma$-aminobutyric acid activity in man: Relationship to age and sex as reflected in CSF. Arch. Neurol. 39, $247-$ 249.

19. McGeer, E. G. (1978) Aging and neurotransmitter metabolism in the human brain. In: Alzheimer's Disease: Senile Dementia and Related Disorders (Katzman, R., Terry, R. D. \& Bick, K. L., eds.) pp. 427-440, Raven Press, New York.

20. Enna, S. J., Stern, L. Z., Wastek, G. J. \& Yamamura, H. I. (1977) Cerebrospinal fluid $\gamma$-aminobutyric acid variations in neurological disorders. Arch. Neurol. 34, 683-685.

21. Hornykiewicz, O., Lloid, K. G. \& Davidson, L. (1976) The GABA, system and function of the basal ganglia and Parkinson's disease. In: GABA in Nervous System Function (Robert, E., Chase, T. N. \& Tower, D. B., eds.) pp. 479485, Raven Press, New York.

Dr. N. Ogawa
Department of Neuroscience
Institute of Molecular
and Cellular Medicine
Okayama University Medical School
2-5-1 Shikata-cho
Okayama 700
Japan

